\title{
A “Crib Sheet" for Supernova Events
}

\author{
David Arnett \\ Steward Observatory, University of Arizona, Tucson AZ 85721, USA \\ ICRA, Rome, Italy
}

\begin{abstract}
This paper summarizes our theoretical understanding of supernova events in a "back of the envelope" way. It is intended to aid in the recognition and understanding of those events which are not "standard", and which may provide the most insight.
\end{abstract}

Keywords: Supernovae

PACS: $95.85-\mathrm{e}, 97.10 . \mathrm{Cv}, 97.10 . \mathrm{Me}, 97.60 .-\mathrm{s}, 97.60 . \mathrm{Bw}$

\section{INTRODUCTION}

This material is abstracted from previous work by the author; see Arnett [1, 2] for a more detailed discussion and references to earlier work. It is hoped that by using the template analysis given below, it will be easier to recognize non-standard events.

\section{A “STANDARD” SUPERNOVA}

We define a standard supernova event ( $\mathrm{SN}$ ) and show how to scale to nonstandard cases. Our standard $\mathrm{SN}$ is assumed to have

- a luminosity of $L=10^{10} \mathrm{~L}_{\odot}$,

- an effective temperature of $T \approx 10^{4} \mathrm{~K}$, and

- will stay bright for a time of a few weeks $\left(2 \times 10^{6} \mathrm{sec}\right)$.

This implies several interesting things:

- Using $L / L_{\odot}=\left(R / R_{\odot}\right)^{2}\left(T_{e} /\left(T_{e}\right)_{\odot}\right)^{4}$, the radius is $R \approx 1.2 \times 10^{15} \mathrm{~cm}$. This is about 20 times larger than the radii of the largest supergiants.

- To move this distance in $2 \times 10^{6} \mathrm{~s}$ implies an average velocity $v \approx 6 \times 10^{8} \mathrm{~cm} / \mathrm{s}$. The photospheric sound speed is only $\sqrt{1.5 R_{\text {gas }} T} \approx 10^{6} \mathrm{~cm} / \mathrm{s}$, so the flow is highly supersonic.

- The energy radiated as visible light is $L t \approx 2 \times 10^{49}$ ergs. Suppose for example, this is fully supplied by radioactive decay of $\mathrm{Ni}^{56}$. At about $2 \mathrm{MeV}$ of plasma heating per decay, this implies more than $0.1 \mathrm{M}_{\odot}$ of that nucleus be freshly produced in the $\mathrm{SN} \sqrt{1}$.

\footnotetext{
${ }^{1}$ Fast production involves a time scale short compared to electron capture time in the dense $\rho>$ $10^{5} \mathrm{~g} / \mathrm{cm}^{3}$ plasma, which is itself much shorter than the half-life of 5.9 days. Further, the time from synthesis to maximum light allows significant $\mathrm{Ni}^{56}$ decay (see [1]).
} 


\section{RADIATIVE DIFFUSION TIME SCALE}

The time scale for radiative diffusion of energy from a sphere of radius $R$ is $\tau_{d}=$ $3 R^{2} / \pi^{2} \lambda c$, where the mean-free-path is $\lambda=1 / \rho \kappa$, with $\rho$ the mass density and $\kappa$ the Rosseland mean opacity. We set this equal to the expansion time scale, $\tau_{e}=R / v$, where $v$ is the velocity of expansion, and of order the average velocity we derived aboved. The average density is $\rho=3 M / 4 \pi R^{3}$, so we obtain a time scale

$$
\tau \approx 3 \times 10^{6} \mathrm{~s}\left[\left(\frac{\kappa}{0.2 \mathrm{~cm}^{2} / \mathrm{g}}\right)\left(\frac{M}{M_{\odot}}\right)\left(\frac{10^{8} \mathrm{~cm} / \mathrm{s}}{v}\right)\right]^{\frac{1}{2}},
$$

which is essentially the time to maximum light for an expanding, diffusing sphere (see $\S 13.3$ in [1]). If the opacity $\kappa$ is near the Thompson scattering value $(0.4$ for pure $\mathrm{H}$ and 0.2 for pure $\mathrm{He}^{4}$ ), that factor is of order unity. Taking $\tau \approx 2 \times 10^{6} \mathrm{~s}$ and $v \approx 6 \times 10^{8} \mathrm{~cm} / \mathrm{s}$, we get $M \approx 2.6 M_{\odot}$ or so. These expressions are approximate; see [1] or recent simulations for more precise values. Nevertheless we see that the mass of the exploding object is star-sized, yet less than the most massive stars (probably due to mass loss).

Using this mass and radius, the average density is $\rho=3 M / 4 \pi R^{3} \approx 10^{-12} \mathrm{~g} / \mathrm{cm}^{3}$, so that for $\kappa=0.2$ the mean-free-path is $\lambda=1 / \rho \kappa \approx 5 \times 10^{12} \mathrm{~cm}$. The object is therefore 240 mean-free-paths deep. This justifies using the diffusion time, but also indicates that supernovae are a transition between optically-thick stars and optically-thin nebulae.

\section{ADIABATIC COOLING AND KINETIC ENERGY}

Taking this mass and the average velocity, the kinetic energy is $K \approx 10^{51} \mathrm{erg}$, or one bethe, so that the radiated photon energy is only about 0.02 of this. Adiabatic cooling converts internal energy into kinetic energy, so that for this radiation-dominated gas, this corresponds to an initial radius smaller by the same factor, 0.02 , which gives $R(0) \approx 2.4 \times 10^{13} \mathrm{~cm}$. This is in the range of radii of red giants. Shock heating can power the light curve only if the progenitor has a radius this large; otherwise the shock energy is degraded into kinetic energy of expansion. To the extent that the light curve is dominated by shock energy, a large radius means a bright, slow event, and a small radius means a dim, fast event.

Mass loss by single massive stars and by binary interactions is an important parameter for interpreting supernovae behavior Arnett [2]. As Eq. 11 indicates, a larger mass gives a slower light curve by increasing the diffusion time?

Unless there is matter to collide with at radii $R>10^{15} \mathrm{~cm}$, the kinetic energy of expansion is unable to provide radiation. How much mass loss would be required to provide enough circumstellar matter to affect the light curve? We consider one interesting case: suppose that the progenitor is a red supergiant losing mass with a velocity $w \approx 10^{6} \mathrm{~cm} / \mathrm{s}$. The uniform mass loss rate for the progenitor would be $d M_{p} / d t=4 \pi r^{2} \rho(r) w$. The

${ }^{2}$ Increased opacity gives a similar effect. 
SN shock would collide with this pre-existing mass distribution, raising the energy of this material at a rate $L_{K}=\left(v^{2} / 2\right) d M_{\text {shock }} / d t$, where the rate of sweeping up of mass is $d M_{\text {shock }}=4 \pi r^{2} \rho(r) v$. Substituting $r^{2} \rho(r)$ we have from the progenitor mass loss, $L_{K}=\left(d M_{p} / d t\right) v^{3} / 2 w$, or

$$
L_{K} / 10^{43} \mathrm{erg} / \mathrm{s} \approx 7.2 \times 10^{2}\left(\frac{d M_{p}}{d t} / \mathrm{M}_{\odot} \mathrm{yr}^{-1}\right) .
$$

A mass loss rate of $10^{-4} \mathrm{M}_{\odot} \mathrm{yr}^{-1}$ from the progenitor in a time $t>R / w \approx 40$ years prior to the explosion implies a significant source of energy. Does it make a visible display? Where does this energy go? The collision occurs in optically-thin plasma, under nonequilibrium conditions; we are in a "nebular" regime. The collision generates turbulence, and that generates magnetic field. Being optically thin, significant radio and $\mathrm{x}$-ray emission occurs. Cosmic-ray acceleration should result (Ginsburg \& Syravotskii [4]). See Fransson, Lundqvist, \& Chevalier [3] for a detailed analysis of the well-observed SN1993J.

\section{RADIOACTIVITY}

Radioactive decay energy cannot be adiabatically cooled until the decay actually happens, so radioactive energy is automatically "saved" until a time corresponding to the mean lifetime of the decay. Each radioactive species adds an exponential decay component to the light curve. For this to be significant, a large amount of mass must be synthesized into a suitable nucleus. $\mathrm{Ni}^{56}$ is exceptional in this regard: it is doubly-magic, and the most bound nucleus having equal numbers of neutron and protons $Z=N$, so it is easy to synthesize by explosions from fuels with $Z=N$. The ashes of all hydrostatic burning stages up to oxygen consumption have $Z=N$ to a suffienctly good approximation to allow profuse production of $\mathrm{Ni}^{56}$. It is the dominant radioactivity found so far. $\mathrm{Ni}^{56}$ decays to $\mathrm{Co}^{56}$ by electron-capture with a 5.9 day halflife. The Co decays by both electron-capture and by positron-emission to $\mathrm{Fe}^{56}$ with a 77.3 day halflife. The characteristic light curves of type I supernovae reflect this double decay, with their "decline from peak" and "tail" 3. More massive ejecta have longer radiative diffusion times, and can smear out the $\mathrm{Ni}^{56}$ peak, leaving only the $\mathrm{Co}^{56}$ tail. The stable product, $\mathrm{Fe}^{56}$, is the sixth most abundant nucleus in the solar-system abundance distribution, and almost all is believed to be formed in supernova explosions.

There are a number of other important radioactive nuclei, such as $\mathrm{Ti}^{44}$ and $\mathrm{Al}^{26}$, which act as tracers of nucleosynthesis but none have yet been found which dominate the light curve so much as the $\mathrm{Ni}^{56}-\mathrm{Co}^{56}-\mathrm{Fe}^{56}$ chain.

\footnotetext{
3 The rise to peak is shaped by radiative diffusion Arnett [1]
} 


\section{LIMITS TO $\mathrm{Ni}^{56}$ PRODUCTION}

Because of its profound affect on light curves, it is interesting to ask if it is possible for such explosions to produce little $\mathrm{Ni}^{56}$. Do all of these explosions have radioactive heating? There are at least two obvious cases in which the $\mathrm{Ni}^{56}$ production is limited:

Density gradient: if the progenitor has a very steep density gradient around the core, there will be be little appropriate fuel. The mass of $\mathrm{Z}=\mathrm{N}$ matter, at right radius to be shocked hot enough to make $\mathrm{Ni}^{56}$, will be too small for much production. This happens for stars with cores very close to the Chandrasekhar mass (e.g., $10 \mathrm{M}_{\odot}$ ).

Accretion: $\mathrm{Ni}^{56}$ is made but collides with a massive mantle, is slowed, and accreted onto a newly formed neutron star or black hole. This happens for fairly massive cores (e.g., stars $35 \mathrm{M}_{\odot}$ on the main sequence, having helium cores of $10 \mathrm{M}_{\odot}$ ).

\section{SOURCES OF LIGHT CURVE ENERGY}

Some possible sources of internal energy to supply supernova light curves:

Shock Breakout The emergence of the supernova shock from the progenitor star. Roughly speaking, this occurs when the photon diffusion time equals the time for the shock to reach the stellar surface.

Diffusion of Shock Energy The leakage of shock-generated heat by radiative diffusion, after the shock has gone beyond the stellar surface (SNII, e.g.).

Diffusion of Heat from Radioactivity The heating by radioactive decay counters adiabatic cooling, and powers the light curve (SNIabc, SN1987A, e.g.)

Heating by New Neutron Star MHD driven by rotational energy (Crab Nebula pulsar), or late accretion (Not recognized?)

Heating by Accretion onto New Black Hole (Not recognized?)

Heating related to Binary Companion (Not recognized?)

Notice that several of these possibilities have not been recognized, and hence might be interesting to look for.

\section{PREDICTION OF UNOBSERVED LIGHT CURVES}

With the rapid advance of astronomical technology, it is interesting to ask if there are types of events that we have not yet seen, but might. In this regard we note that what are now called supernova of Type Ib and Ic, were suggested to exist before they were recognized; they were the "bare cores" discussed in Arnett [2]. Elias, et al. [5] suggested that their observational data on 11 Type I supernovae fell into two classifications, Ia and $\mathrm{Ib}$. The brighter Ia's correspond to exploding white dwarf stars, for which analytic solutions for the light curves were available Arnett [6]. The dimmer Ib's correspond to core collapse events which produce $\mathrm{Ni}^{56}$, which Colgate \& McKee [7] first introduced to explain all types of supernovae. Arnett [2] had suggested that both types should be found, and that the core collapse events would be dimmer because they would tend to 
TABLE 1. SN Parameters and Light Curves

\begin{tabular}{lrrrrr}
\hline$R(0)$ & $M_{e j}$ & Radioactivity & Brightness & Speed & Example \\
\hline small & small & large & bright & fast & SNIa \\
large & large & some & medium & slow & SNIIP \\
large & small & some & medium & slow & SNIIL \\
small & small & some & medium & fast & SNIb,c* \\
small & large & some & medium & slow & SN1987A \\
large & small & some & medium & fast & SN1993J \\
large & small & no & medium & fast $t^{\dagger}$ & \\
small & small & no & dim & fast & \\
large & large & no & dim & slow & \\
\hline & & & & \\
* Predicted in Arnett [2] & & & & \\
shock & & & &
\end{tabular}

make less $\mathrm{Ni}^{56}$. Filippenko, Porter, \& Sargent [8] found a further class, Ic's, which unlike Ib's, are He poor; apparently they are bare cores that lost He as well as H. Wheeler \& Harkness [9] give a review of the observational situation at the time.

\section{A Table of Some Possibilities}

Table 1 sketches the sorts of exploding supernovae we might expect to find; other entries may need to be added. The parameters varied here, besides initial mass, are initial radius $R(0)$, mass ejected by explosion $M_{e j}$, and presence of radioactivity. Even this limited set covers much of the observed data set, at least in a crude way. But is the limited set of paramaters sufficient for a more complete and accurate data set?

\section{SOME FINAL COMMENTS}

Some key points:

- It is not theoretically demanded that all "supernova" explosions have radioactive ejecta and/or large initial radii, the two features that make them bright. Therefore, explosions of small initial radii and little radioacitity in their ejecta would be underrepresented in the present data set. Such events would be dim and fast.

- None of these entries are related to binary interactions except is a fairly passive way of slow mass transfer for SNIa's. Are there merger supernovae (for example two white dwarfs or a white dwarf and a neutron star or black hole)?

- Mass loss, either by winds from single stars or by binary interactions, is an importent parameter for the observed explosion; mass loss is not well understood from a predictive basis yet.

- Rotation and magnetic fields provide vector fields which interact and which we are just beginning to learn to simulate plausibly; they are expected to be important 
aspects of the understanding of the formation of relativistic jets and GRB's, as well as the general issue of angular momentum transport in stars.

- Fully 3D simulations of oxygen and silicon burning are in their infancy (Meakin $\&$ Arnett [10, 11]), and already there are indications of complexity we have not anticipated. For example, 2D simulations of $\mathrm{C}, \mathrm{Ne}, \mathrm{O}$, and $\mathrm{Si}$ shells indicate that there are interactions between burning shells which are mediated by waves (p-, g-, and mixed-modes), and none of this has yet been put into evolutionary models.

- Extrapolation to earlier cosmological epochs is made more uncertain by a lack of fundamental understanding of how magnetic fields, rotation, binary formation, and mass loss change with metalicity, yet interpretation of some of the new supernova observations may be affected by these issues.

Despite these theoretical challenges, the prospect for observation of previously inaccessible phenomena is excellent. For supernova studies, the exploration of a broad time domain and greater completeness is exciting. It would be both a surprise and a disappointment if some really new phenomena, either unrecognized or unexpected, do not appear soon!

\section{ACKNOWLEDGMENTS}

This work was supported in part by NSF Grant 0708871 and NASA Grant NNX08AH19G at the University of Arizona. The kind hospitality of Prof. Sandip K. Chakrabarti and the Bose Center for Fundamental Research is gratefully acknowledged.

\section{REFERENCES}

1. D. Arnett, Supernovae and Nucleosynthesis, Princeton University Press, Princeton, NJ, 1996, pp. 414 457.

2. D. Arnett, Eighth Texas Symposium on Relativistic Astrophysics, ed. M. D. Papagiannis, Annals of the New York Academy of Sciences, Vol. 302, New York, NY, 1977, pp. 90-100.

3. Frasson, C., Lundqvist, P., \& Chevalier, R., 1996, Ap. J.,461, 993

4. V. L. Ginsburg \& S. I. Syrovatskii, The Origin of Cosmic Rays, 1964, Oxford, Pergammon Press

5. Elias, J. H., Mathews, K., Neugebauer, G., \& Persson, S. E., 1985, Ap. J., 296, 379

6. Arnett, W. D., 1982, Ap. J., 253, 785

7. Colgate, S. A., \& McKee, C., 1969, Ap. J., 157, 623

8. Filippenko, A. V., Porter, A. C., \& Sargent, W. L. W., 1990, A. J., 100, 1575

9. Wheeler, J. C., \& Harkness, R. P., 1990, Rep. Prog. Phys., 53, 1467

10. Meakin, C., \& Arnett, D., 2007, Ap. J., 667, 448

11. Meakin, C., \& Arnett, D., 2006, Ap. J., 637, 53 